\title{
Improved picoliter-sized micro-reactors for high-throughput biological analysis
}

\author{
HAN WeiJing ${ }^{1,3 \dagger}$, YUAN LiNa ${ }^{2,3 \dagger}$, WEI QingQuan ${ }^{1,3}$, LI YunTao ${ }^{1,3}$, REN LuFeng $^{2,3}$, \\ ZHOU XiaoGuang ${ }^{1,3}$, YU Jun ${ }^{2,3 *} \&$ YU YuDe Y, $^{1,3}$ \\ ${ }^{1}$ State Key Laboratory of Integrated Optoelectronics, Institute of Semiconductors, Chinese Academy of Sciences, Beijing 100083, China; \\ ${ }^{2}$ DNA Sequencing Technologies R\&D Center, Beijing Institute of Genomics, Chinese Academy of Sciences, Beijing 100101, China; \\ ${ }^{3}$ The Joint Laboratory of Bioinformation Acquisition and Sensing Technology, Institute of Semiconductors, Beijing Institute of Genomics, \\ Chinese Academy of Sciences, Beijing 100083, China
}

Received June 16, 2013; accepted August 19, 2013

\begin{abstract}
High-throughput pyrosequencing, carried out in millions of picoliter-sized reactors on a fiber-optic slide, is known for its longer read length. However, both optical crosstalk (which reduces the signal-to-noise ratio of CCD images) and chemical retention adversely affect the accuracy of chemiluminescence determination, and ultimately decrease the read length and the accuracy of pyrosequencing results. In this study, both titanium and oxidized aluminum films were deposited on the side walls and upper faces of micro-reactor slides to enhance optical isolation; the films reduced the inter-well crosstalk by one order of magnitude. Subsequently, chemical retention was shown to be caused by the lower diffusion coefficient of the side walls of the picolitersized reactors because of surface roughness and random pores. Optically isolated fiber-optic slides over-coated with silicon oxide showed smoother surface morphology, resulting in little chemical retention; this was further confirmed with theoretical calculations. Picoliter-sized micro-reactors coated with titanium-silicon oxide films showed the least inter-well optical crosstalk and chemical retention; these properties are expected to greatly improve the high-throughput pyrosequencing performance.
\end{abstract}

high throughput analysis, picoliter-sized micro-reactor, surface coating

Citation: Han W J, Yuan L N, Wei Q Q, et al. Improved picoliter-sized micro-reactors for high-throughput biological analysis. Sci China Life Sci, 2013, 56: 1134-1141, doi: 10.1007/s11427-013-4564-3

Genomic sequencing has garnered great attention and experienced tremendous development in recent years. Prior to this "explosion", conventional Sanger sequencing had been used to obtain a wide range of genomic information, from bacteria to humans [1]. However, the reliance on capillary electrophoresis means that conventional sequencing is not likely to become a particularly low-cost, high-speed, or high-throughput technique. The advent of next-generation sequencing, based on massively parallel arrays of individual sequencing reactions, has drastically improved the se-

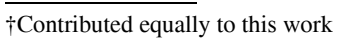

*Corresponding author (email: yudeyu@ semi.ac.cn; junyu@big.ac.cn) quencing throughput and greatly reduced cost and time [2-7]. High-throughput pyrosequencing [8], relying on detection of chemiluminescence signals generated from millions of DNA polymerization reactions, has shown excellent accuracy, with longer read lengths than those available with other next-generation technologies, and has been successfully used for de novo sequencing, resequencing, and metagenomic sequencing [9-13].

High-throughput pyrosequencing has been achieved through technological advances in two major areas: the introduction of solid-supports for the sequencing reaction $[8,14,15]$ and the application of etched fiber-optic slides for optical coupling $[8,14,15]$. The former includes beads to 
support DNA during amplification through emulsion PCR for template library preparation and to carry the enzymes required for sequencing to avoid the requirement for the replenishment of enzymes in flow conditions [15]. Etched fiber-optic slides provide picoliter-sized micro-reactors for high density parallel reactions and transfer millions of photons for CCD detection in situ. High-throughput pyrosequencing is accomplished by injecting fresh reagents into micro-wells via a laminar flow stream, recording chemiluminescence signals in real-time, and washing away both byproducts and unincorporated reagents before the next nucleotide incorporation $[14,15]$. Optically-addressable fiber-optic slides [16-18] with millions of picoliter-sized vessels have been designed to accomplish high density reactions and also serve as monolithic substrates to satisfactorily transmit the parallel chemiluminescence photon individually and simultaneously with low light loss. Precise determination of light intensity of each reaction is critical to ensure the accurate count of nucleotides incorporated during the homopolymer run; the precision can be reduced in two ways. First, optical crosstalk between adjacent micro-wells reduces the signal-to-noise ratio and affects the accuracy of light intensity determination for each individual reaction. Second, chemical residues carried over from one sequencing cycle to the next in the picoliter-sized reactors result in chemiluminescent interference and introduce strong background noise. Chemical retention, especially retention of deoxyribonucleotides (dNTP), also causes loss of synchronicity in an individual vessel, which then affects the read length. The construction of picoliter-sized reactors with enhanced optical isolation and reduced chemical retention will greatly promote the signal-to-noise characteristics of the micro-reactor slides.

Here, we studied the effects of picoliter-sized microreactor properties on two factors affecting the signal-tonoise ratio for high-throughput pyrosequencing: the optical isolation of reaction wells and surface retention of chemical residues. Furthermore, a theoretical model was constructed to examine the effects of surface properties of reactors on chemical retention.

\section{Materials and methods}

\subsection{Fabrication of picoliter-sized reactors}

Fiber-optic slides with the dimensions $48.9 \mathrm{~mm} \times 47.9 \mathrm{~mm}$ $\times 2 \mathrm{~mm}$ were fabricated to fit flow cells of the BIGIS-4 sequencer [19]. Individual micro-reactors were formed by anisotropic etching of optic fibers on one side of the slide with a mixture of hydrofluoric acid, ammonium fluoride, and deionized water at room temperature [20-22]. Anisotropic etching of the cladding and core of the fibers created one million micro-wells, each $28 \mu \mathrm{m}$ in diameter and surrounded by $6 \mu \mathrm{m}$ cladding, on the fiber-optic slide; the etching depth of the micro-wells was $30 \mu \mathrm{m}$ with a cen- ter-to-center pitch of $34 \mu \mathrm{m}$ (Figure S1 in Supporting Information), resulting in a calculated well size of $20 \mathrm{pL}$ and a well density of 1000 wells $\mathrm{mm}^{-2}$. The surface of the unetched side of the fiber-optic slide was polished to prevent optical scattering when directly coupled to the plate face of the CCD.

\subsection{Surface coating}

Titanium and aluminum metal films were deposited on the cladding of the micro-wells to enhance optical isolation. An angled evaporation plant [23] was designed to achieve selective deposition and installed in an electron beam evaporation chamber (Innotec, USA). Fiber-optic slides were mounted onto the plant; the surface of the slides and that of instrument mount formed an angle $\theta$, which needed to be varied depending on the aspect ratio of the micro-wells. The evaporation plant was adjusted to an angle such that a metal film deposited on the side walls but not the bottom of the micro-wells.

To achieve optical isolation for visible light, we deposited $100 \mathrm{~nm}$ titanium or $50 \mathrm{~nm}$ aluminum films on the walls of the micro-wells; the thickness of the metallic film was chosen based on the skin depth for visible light. For aluminum, fiber-optic slides were subsequently placed in an oxygen environment to form an aluminum oxide layer.

To minimize the surface affinity for molecules from the solution, we over-coated micro-wells with an additional silicon oxide dielectric layer $200 \mathrm{~nm}$ thick, by magnetron sputtering [24-26], forming titanium-silicon oxide coated and aluminum-silicon oxide coated fiber-optic slides. The surface morphologies of the four different coatings were examined by atomic force microscopy (AFM).

\subsection{Performance of surface coated fiber-optic slides}

\subsubsection{Optical characterization}

Polystyrene beads coated with the fluorophore Cy3 (excitation wavelength maximum at $550 \mathrm{~nm}$, emission maximum at 568-574 $\mathrm{nm}$ ) were used to simulate chemiluminescence during pyrosequencing [27] and thus examine the optical characteristics of the reaction slides. Bare polystyrene beads, $25 \mu \mathrm{m}$ in diameter, were obtained from Kisker Biotech GmbH \& Co. KG, Germany; poly(phe-lys), glutaraldehyde, and Cy3-streptavidin were purchased from Sigma-Aldrich. Cy3-coated polystyrene beads were prepared as follows: the surfaces of bare polystyrene beads were modified with poly(phe-lys): glutaraldehyde was covalently bound to the poly(phe-lys), and finally, Cy3-streptavidin was coupled to the surface of the polystyrene microbeads by a condensation reaction.

Cy3-coated polystyrene beads were loaded into the picoliter-sized micro-reactors of uncoated, metallic coated, and silicon oxide over-coated reaction slides by centrifugation. After loading of beads, the four differently-coated reaction 
slides were examined under a fluorescence microscope from both sides of the slides to study the optical transmission and isolation characteristics. Fluorescence images obtained from an Olympus fluorescence microscope were analyzed by the ImageJ software package to calculate the light intensity distributions in the micro-wells.

\subsubsection{Chemical retention characterization}

DNA beads covered with 10 million copies of a unique and known DNA template, allowing for a synchronous sequencing signal, were used to examine the chemical retention of the four differently-coated fiber-optic slides. Library preparation and amplification were carried out according to the Rapid Library Preparation Method and emPCR Amplification Method manuals of the 454 sequencing system [8]. After emulsion breaking and selective enrichment, sequencing primer was annealed to the clonally amplified DNA fragments on the DNA beads. The template-carrying DNA beads were incubated with Bst DNA polymerase, apyrase, and single-stranded binding protein, and then loaded into the micro-wells of the fiber-optic slides by centrifugation along with enzyme beads (ATP-sulfurylase and luciferase) and packing beads.

The packed fiber-optic slide was installed in one of the four flow cells of the BIGIS-4 sequencer, with the polished surface coupled directly against the fiber-optic faceplate of the CCD camera [19]. Sequencing reagents, including dNTP, D-luciferin, and adenosine phosphosulfate (APS), were pumped into the flow-cell. dNTPs were sequentially introduced in the order of T, A, C, and G. Incorporation of dNTPs resulted in the release of inorganic pyrophosphate (PPi), triggering an ATP-sulfurylase catalyzed reaction to generate the energy needed for the luciferase-catalyzed oxidation reaction to give rise to chemiluminescence which was recorded by the CCD camera. A wash step was followed to remove residual nucleotides, $\mathrm{PPi}$, and $\mathrm{ATP}$ in preparation for the next nucleotide injection cycle (Figure S2).

\section{Results and discussion}

\subsection{Optical characteristics of reaction slides}

Picoliter-sized micro-reactors on fiber-optic slides made possible simultaneous observation of millions of independent reactions for high-throughput pyrosequencing. Chemiluminescence signals from each micro reaction were recorded by a highly-sensitive CCD camera. The precise determination of the light intensity from each well was important for accurately identifying the nucleotides incorporated during homopolymer runs. Inter-well optical crosstalk reduced the signal to noise ratio of the raw image, and affected the accuracy of the determination of the light intensity from each well.

We used Cy3-coated polystyrene micro-beads as a model system to study the optical characteristics of the sequencing slide. A representative fluorescence image of an uncoated fiber-optic slide captured from the unetched side is shown in Figure 1A. The image is consistent with the image obtained from the etched side of the slide (data not shown), confirming that the fiber-optic slide transmitted light very well. However, silica cladding of the micro-wells did not prevent the crosstalk of the fluorescence signal into adjacent micro-wells, which caused the boundaries between wells to become less clearly defined. This optical crosstalk was further quantified by calculating the ratio of the crossover-light intensity to the total light intensity from a selected well (the blue box in Figure 1A) and was shown in the 3D fluorescence distribution; a portion of the fluorescent light passed through the silica cladding and entered the neighboring picoliter-sized reactors (Figure 1B). The optical crosstalk calculated from multiple locations ranged between $25 \%$ and $35 \%$ (Figure 1C).

To eliminate the optical crosstalk between micro-wells, we investigated the effect of coating of the silica cladding. Dielectric films and metallic films are commonly used to reduce the light transmission of optical devices [28]. Dielectric films can achieve extremely low transmittance but their bandwidth is too narrow for chemiluminescence. Metallic films, with their distinct opacity toward visible light and adequate bandwidth across the entire visible spectrum, afford a better alternative. In this work, low-cost titanium and aluminum (with oxidized layer) were chosen for their biocompatible properties [29-32].

Oxidized aluminum and titanium coatings were deposited on the side walls and the upper faces of the microreactors by a reformation vacuum coating process. Fluorescent Cy3-coated polystyrene beads were loaded into coated micro-wells to examine the optical characteristics of the wells. Fluorescence images of oxidized aluminum-coated and titanium-coated fiber-optic slides were obtained from the polished side and shown in Figure 1D and G, respectively. Images from the etched side were also taken (not shown) and exhibited very similar light intensities, confirming that the metallic coating process on the side walls did not affect the light transmission through the bottom of fiber-optic slide. Inter-well optical leakage was dramatically reduced in comparison with the uncoated image (Figure 1A). The 3D fluorescence intensity distributions of aluminum oxide- and titanium-coated reaction vessels (taken from the micro-wells in the blue boxes shown in Figure 1D and G) showed that no obvious fluorescence leakage could be detected (Figure 1E and $\mathrm{H}$ ). Examination of hundreds of individual wells showed an average light crosstalk of $1 \%$ for the aluminum oxide-coated fiber-optic slide (Figure 1F) and $3.5 \%$ for the titanium-coated fiber-optic slide (Figure 1I). This shows that the metallic coating of the fiber-optic slides has effectively eliminated any optical crosstalk, and that the slides now exhibit optical properties suitable for parallel chemiluminescence signal detection. In addition, the optical characteristics (not shown) of the fiber-optic slides over- 


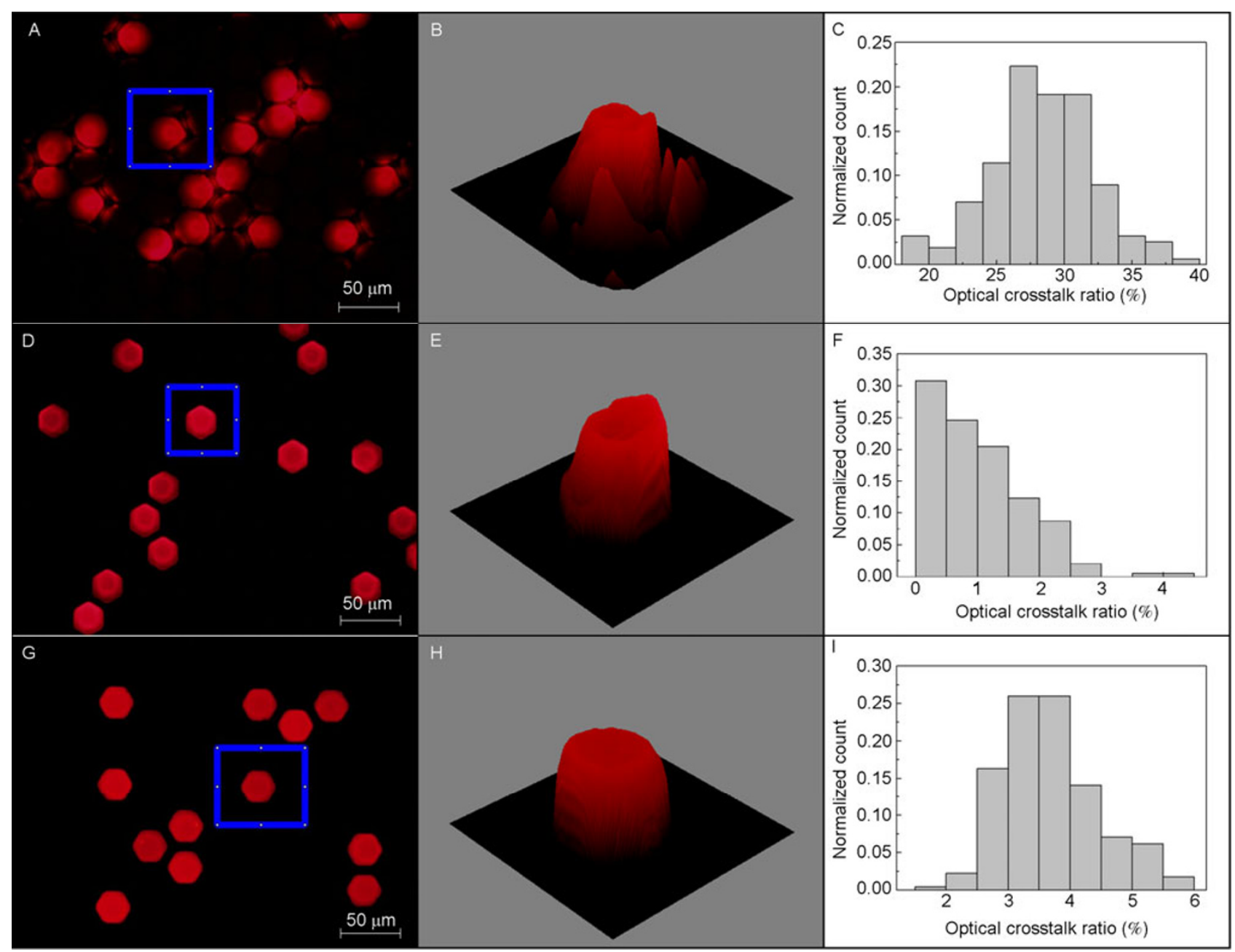

Figure 1 Optical properties of differently coated fiber-optic slides. From top to bottom, each row shows data for uncoated, oxidized aluminum-coated, and titanium-coated fiber-optic slides; from left to right, columns are fluorescence images, three-dimensional fluorescence distributions (from the regions shown in the blue boxes from the first column), and statistical distributions of optical crosstalk. Optical leakage from the fiber-optic slides with metallic coating was dramatically reduced in comparison with that of the uncoated slide.

coated with an additional silicon oxide dielectric layer were consistent with those of the metallic-coated slides.

\subsection{Chemical residues}

High-throughput pyrosequencing is accomplished by automated injection of sequencing reagents into an array of micro-wells simultaneously using a laminar flow stream $[14,15]$. After each base extension, a wash step is needed to remove unincorporated nucleotides and reaction byproducts (Flow field simulation in Supporting Information) because accumulation of chemical residues in reaction vessels inhibits polymerase activity, contributes to signal background, and reduces the optical contrast.

Four differently coated fiber-optic slides loaded with DNA beads, enzyme beads, and packing beads were installed in four independent flow cells of the BIGIS-4 sequencer. Reaction substrates and repeated series of dTTP, dATP, dCTP, and dGTP were pumped into the flow cells.
After chemiluminescence imaging, residues of unincorporated dNTP, ATP, and PPi in picoliter reactors were washed away with sequencing buffer to eliminate interference with the next sequencing cycle. Chemiluminescence images of expanded regions of the first successful base incorporation with the four differently coated fiber-optic slides are shown in Figure 2A-D. Each nine-pixel square represents the signal emitted from one reaction vessel. After the wash step, images from all four fiber-optic slides showed backgrounds consistent with the electronic noise of the CCD (not shown). When the next unmatched base was pumped into the flow cells, however, chemiluminescence was observed for both titanium and oxidized aluminum coated fiber-optic slides (Figure 2E and F), while silicon oxide over-coated fiber-optic slides showed little residual signal (Figure 2G and $\mathrm{H})$. The unexpected chemiluminescence was the result of residual chemicals, e.g., PPi, on the slide surface from the previous sequencing cycle. This suggests that the silicon oxide-modified surface promoted better removal of unin- 


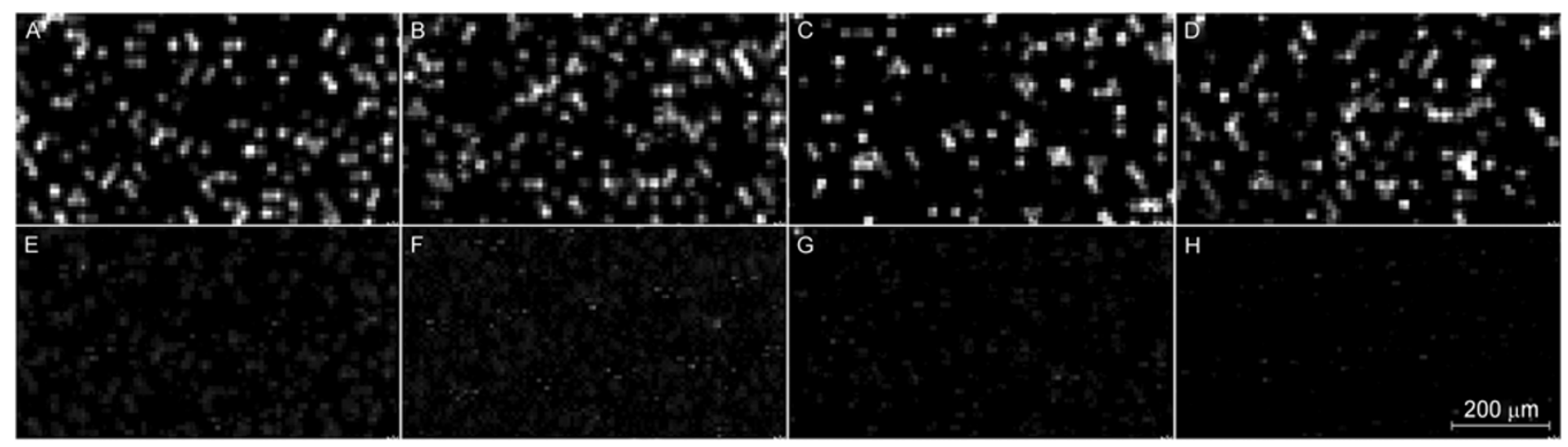

Figure 2 Chemiluminescence images of pyrosequencing in differently coated micro-reactors. From left to right, columns show oxidized aluminum-coated, titanium-coated, oxidized aluminum-silicon oxide coated, and titanium-silicon oxide coated fiber-optic slides; from top to bottom, rows are base incorporated images and non-base incorporated images; all figures share the scales shown in Figure $2 \mathrm{H}$. Metallic coated fiber-optic slides showed unexpected chemiluminescence, while silicon oxide over-coated ones effectively avoided chemiluminescent interference; the titanium-silicon oxide coated fiber-optic slide showed the least background signal.

corporated nucleotides and reaction byproducts from the picoliter reactors by washing buffer.

We further examined the effect of four different kinds of coated surfaces on the diffusion of unincorporated nucleotides and byproducts. The influence of physical surface properties and microstructures on microfluidic flow has been previously studied [33-36]. Here, both theoretical and experimental data showed that the surface morphology, including surface roughness and pores, has a great impact on the fluid movement, especially, the diffusion coefficient. Generally, the diffusion coefficient D on a surface can be described as [37-39]

$$
D=a^{2} / 4 \tau,
$$

where $a$ is the displacement of a molecule traveling in the fluid, and $\tau$ is a finite time interval that a fluid particle diffuses between consecutive hops, defined through the Arrhenius formula:

$$
\tau=A \mathrm{e}^{E / \beta},
$$

where $E$ denotes the energy gained because of the motion in the direction of the van der Waals forces and the energy lost by breaking of the bonds with several fluid molecules, $\beta$ is the product of the Boltzmann constant and temperature, and $A$ depends on the pathway taken by the molecules, which, in turn, is related to the physical morphology of the surface. The relationship between diffusion coefficient and surface morphology can be established by substituting eq. (2) into (1):

$$
D=a^{2} \mathrm{e}^{-\mathrm{E} / \beta} / 4 A .
$$

Eq. (3) shows that the diffusion coefficient decreases with an increase in the number of pathway with the same displacement.

We suspected that the surfaces over-coated with silicon oxide were much smoother than those that had simply been metallically coated, and therefore realized faster diffusion and exhibited higher diffusion coefficients. Surface morphological parameters of four different coatings were obtained by AFM and diffusion coefficients were calculated with the diffusion coefficient equation. The distribution of the diffusion coefficient ratio, defined as the ratio of the diffusion coefficient of a coated surface and the diffusion coefficient of an ideal plane, was used to compare the diffusion properties of different coatings. The AFM images of metallic coatings exhibited uneven and rough surfaces with many random pores (Figure $3 \mathrm{~A}$ and $\mathrm{B}$ ). The distributions of diffusion coefficient ratios of oxidized aluminum and titanium films are shown in Figure $3 \mathrm{E}$ and $\mathrm{F}$, and the average diffusion coefficient ratios were calculated to be 0.13 and 0.16 , respectively. This confirmed that surface roughness and random pores significantly increased the number of pathways for molecules to move the same displacement, resulting in decreased diffusion velocity and diffusion coefficient. Over-coating with silicon oxide, however, made surfaces smother and significantly reduced surface roughness and pores (Figure 3C and D). Distribution of diffusion coefficient ratios of silicon oxide over-coated oxidized aluminum and titanium films are shown in Figure $3 \mathrm{G}$ and $\mathrm{H}$; the average diffusion coefficient ratios increased to 0.35 and 0.5 , respectively.

To further determine the impact of diffusion on chemiluminescent interference, we constructed a model to simulate the process of pyrosequencing in micro-wells. In pyrosequencing, chemiluminescent interference could come from either PPi or ATP produced as part of the pyrophosphate chain reaction. However, the concentration of ATP was about $1 / 10$ of that of PPi [14] and ATP was easily hydrolyzed to adenosine diphosphate (ADP) and inorganic phosphate. The images taken immediately following the wash step confirmed that there was little ATP remaining in the micro-wells. Therefore, PPi residues were the major cause of background chemiluminescence. The NavierStokes equation at steady state, continuity equation, and a 


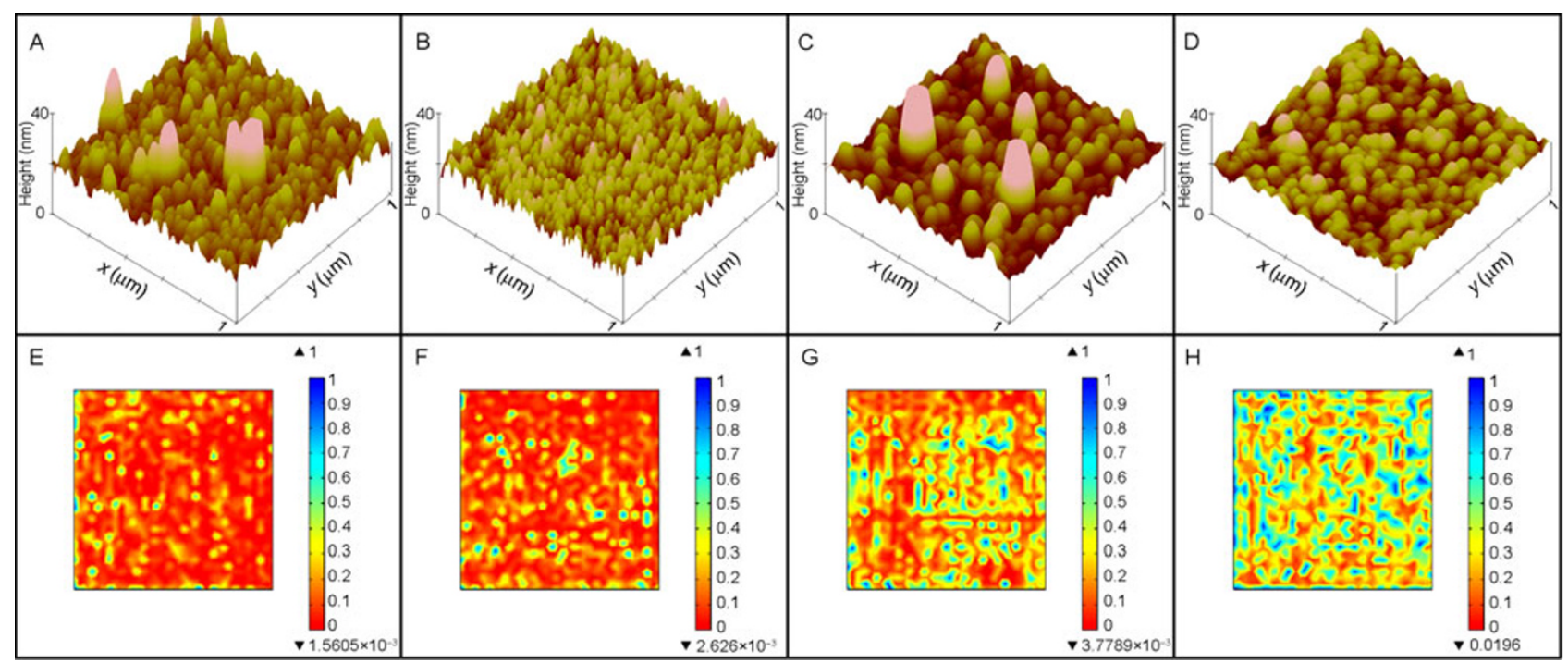

Figure 3 AFM images of surface morphology (upper) and diffusion coefficient ratios (lower) of four different coatings. From left to right, columns represent oxidized aluminum, titanium, oxidized aluminum-silicon oxide and titanium-silicon oxide films. Metallic films with rough surfaces and a significant number of randomly-shaped pores showed extremely low diffusion coefficients through the AFM scanning area; after over coating with silicon oxide, surface roughness and pores were reduced dramatically, and the diffusion coefficient increased correspondingly.

general conservation equation incorporating bulk reaction terms and convection-diffusion equations for transport were combined to describe the PPi behavior in micro-wells and to study the effect of surface roughness on diffusion properties (Individual well diffusion of PPi with different diffusion coefficient in Supporting Information). The calculation was based on the assumption of 20 million DNA fragments per bead, generating an initial concentration of $1.5 \mu \mathrm{mol} \mathrm{L}^{-1}$ of unextended DNA fragments $[8,14]$.

The simulations showed that a higher diffusion coefficient results in fewer chemical residues. PPi generated in the process of DNA polymerization was consumed in the ATP-sulfurylase catalytic process. The traces of PPi concentration in the four differently coated micro-wells are shown in Figure 4. After each sequencing cycle and wash step with interval $t_{0}$, very little PPi residue remained in the titanium-silicon oxide coated picoliter-sized micro-reactors, while $4 \%$ of PPi remained in the oxidized aluminum-silicon oxide coated micro-reactors, and more than $15 \%$ in the micro-reactors coated with metallic films (Figure 4). This last amount is enough to cause serious chemiluminescent interference and reduce the signal-to-noise ratio of subsequent sequencing reactions. Although residues in micro-wells can be reduced by increasing wash time, prolonging the wash step will limit the speed of the sequencing cycles.

Moreover, the diffusion coefficient also affected the peak time and peak height of PPi accumulation during the high-throughput pyrosequencing. The DNA polymerization process was affected by the diffusion velocity of dNTPs, which was directly proportional to the diffusion coefficient. The titanium-silicon oxide coated micro-well with the highest diffusion coefficient showed the fastest PPi accumulation, which peaked $2 \mathrm{~s}$ after pyrosequencing initiation

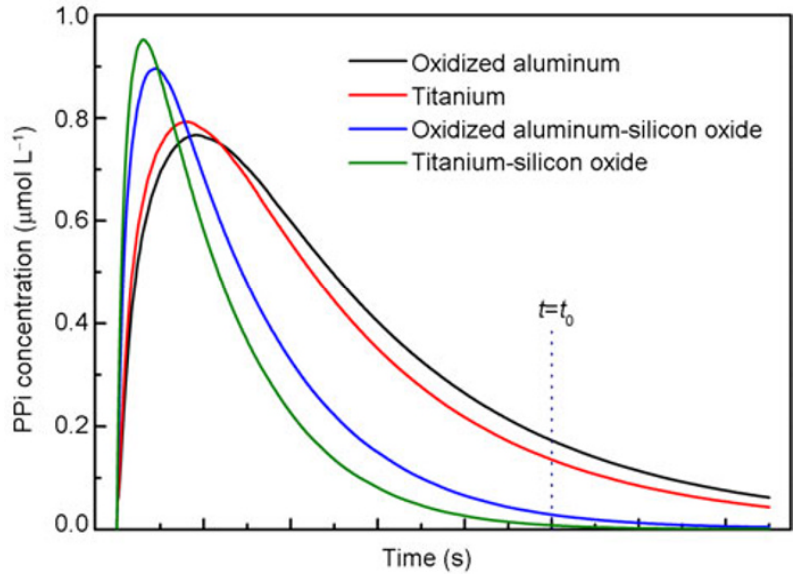

Figure 4 Traces of PPi concentration during pyrosequencing reaction in differently coated micro-wells. Titanium-silicon oxide coated micro-wells showed the fastest and narrowest time profile, in agreement with previous reports. For the three coated slides, the PPi peak was delayed and the peak height lowered by the longer diffusion process.

(Figure 4), and the peak height was $2 / 3$ of that expected from the initial concentration of unextented DNA fragments. This was in agreement with the previous report [14]. However, for the other three coated vessels, the peak of the PPi concentration profile was delayed and broadened, because of prolonged diffusion (Figure 4).

In conclusion, the theoretical simulation was in good agreement with the experimental results, confirming that the titanium-silicon oxide coated micro-wells exhibited the best diffusion characteristics and gave the lowest chemical retention. This increased the signal-to-noise ratio of the raw image, thus improving the read length and accuracy of high throughput pyrosequencing. 


\section{Conclusion}

Picoliter-sized micro-reactor slide directly coupled to the CCD camera enables millions of parallel chemical reactions to be monitored simultaneously. The physical properties of the reactors, especially their optical characteristics and surface morphology, are crucial to the performance of the sequencing system. In this work, the biocompatible metals, titanium and aluminum were selectively deposited on the side walls and upper face of picoliter-sized vessels to promote optical isolation and reduce optical crosstalk. The metal coatings were then over-coated with silicon oxide to provide a smoother surface and thus reduce unwanted chemical retention during the pyrosequencing cycle. Silicon oxide over-coated slides demonstrated not only excellent optical isolation but also smoother surfaces, effectively reducing chemical retention. Picoliter-sized micro-reactors coated with titanium and over-coated with silicon oxide showed the best overall performance and possessed optical and chemical properties suitable for high-throughput pyrosequencing.

This work was supported by the Scientific Equipment Research Project of Chinese Academy of Sciences (YZ200823).

1 Sanger F, Nicklen S, Coulson A R. DNA sequencing with chain-terminating inhibitors. Proc Natl Acad Sci USA, 1977, 74: 5463-5467

2 Bains W, Smith G C. A novel method for nucleic acid sequence determination. J Theoret Biol, 1988, 135: 303-307

3 Drmanac R, Labat I, Brukner I, et al. Sequencing of megabase plus DNA by hybridization: Theory of the method. Genomics, 1989, 4: 114-128

4 Khrapko K R, Lysov Yu P, Khorlyn A A, et al. An oligonucleotide hybridization approach to DNA sequencing. FEBS Lett, 1989, 256: 118-122

5 Ronaghi M, Karamohamed S, Pettersson B, et al. Real-time DNA sequencing using detection of pyrophosphate release. Anal Biochem, 1996, 242: 84-89

6 Ronaghi M, Uhlen M, Nyren P. A sequencing method based on real-time pyrophosphate. Science, 1998, 281: 363-365

7 Brenner S, Williams S R, Vermaas E H, et al. In vitro cloning of complex mixtures of DNA on microbeads: Physical separation of differentially expressed cDNAs. Proc Natl Acad Sci USA, 2000, 97 : $1665-1670$

8 Margulies M, Egholm M, Altman W E. Genome sequencing in microfabricated high-density picolitre reactors. Nature, 2005, 437: 376-380

9 Meyer E, Aglyamova G V, Wang S, et al. Sequencing and de novo analysis of a coral larval transcriptome using 454 GSFlx. BMC Genomics, 2009, 10: 219-236

10 Ley T J, Mardis E R, Ding L, et al. DNA sequencing of a cytogenetically normal acute myeloid leukaemia genome. Nature, 2008, 456: 66-72

11 Li R Q, Fan W, Tian G, et al. The sequence and de novo assembly of the giant panda genome. Nature, 2010, 463: 311-317

12 Krober M, Bekel T, Diaz N N, et al. Phylogenetic characterization of a biogas plant microbial community integrating clone library 16S-rDNA sequences and metagenome sequence data obtained by 454-pyrosequencing. J Biotechnol, 2009, 142: 38-49

13 Petrosino J F, Highlander S, Luna R A, et al. Metagenomic pyrose- quencing and microbial identification. Clin Chem, 2009, 55: 856-866

14 Leamon J H, Rothberg J M. Cramming more sequencing reactions onto microreactor chips. Chem Rev, 2007, 107: 3367-3376

15 Rothberg J M, Leamon J H. The development and impact of 454 sequencing. Nat Biotechnol, 2008, 26: 1117-1124

16 Walt D R. Fibre optic microarrays. Chem Soc Rev, 2010, 39: 38-50

17 Epstein J R, Leung P K, Lee $\mathrm{K} \mathrm{H}$, et al. High-density, microsphere-based fiber optic DNA microarrays. Biosens Bioelectron, 2003, 18: 541-546

18 Pantano P, Walt D R. Analytical applications of optical imaging fibers. Anal Chem, 1995, 65: 481-487

19 Yuan L N, Ren L F, Li Y T, et al. A complete genome assembly of Glaciecola mesophila sp. nov. sequenced by using BIGIS-4 sequencer system. Sci China Life Sci, 2011, 54: 835-840

20 Pantano P, Walt D R. Ordered nanowell arrays. Chem Mater, 1996, 8: 2832-2835

21 Pangaribuan T, Yamada K, Jiang S D, et al. Reproducible fabrication technique of nonametric tip diameter fiber probe for photon scanning tunneling microscope. Jpn J Appl Phys, 1992, 31: 1302-1304

22 Leamon J H, Lee W L, Tartaro K R, et al. A massively parallel PicoTiterPlate based platform for discrete picoliter-scale polymerase chain reactions. Electrophoresis, 2003, 24: 3769-3777

23 Han W J, Wei Q Q, Li Y T, et al. Fabrication of SU8-based chip suitable for genomic sequencing. Acta Phys Sin, 2013, 14: 148701

24 Shoffner M A, Cheng J, Hvichia G E, et al. Chip PCR. I. Surface passivation of microfabricated silicon-glass chips for PCR. Nucl Acids Res, 1996, 24: 375-379

25 Wilding P, Kricka L J, Cheng J, et al. Integrated cell isolation and polymerase chain reaction analysis using silicon microfilter chambers. Anal Biochem, 1998, 257: 95-100

26 Taylor T B, Winn-Deen E S, Picozza E, et al. Optimization of the performance of the polymerase chain reaction in silicon-based microstructures. Nucl Acids Res, 1997, 25: 3164-3168

27 Ronaghi M. Pyrosequencing sheds light on DNA sequencing. Genome Res, 2001, 11: 3-11

28 Macleod H A. Thin-film Optical Filters. London: Institute of Physics Publishing, 1986

29 Li J X, Zhao Y M. Biocompatibility and antibacterial performance of titanium by surface treatment. J Coat Technol Res, 2012, 9: 223-228

30 Lin C C, Cheng H C, Huang C F, et al. Enhancement of biocompatibility on bioactive titanium surface by low-temperature plasma treatment. Jpn J Appl Phys, 2005, 44: 8590-8598

31 La Flammea K E, Popatb K C, Leonic L, et al. Biocompatibility of nanoporous alumina membranes for immunoisolation. Biomaterials, 2007, 28: 2638-2645

32 Tercero J E, Namin S, Lahiri D, et al. Effect of carbon nanotube and aluminum oxide addition on plasma-sprayed hydroxyapatite coating's mechanical properties and biocompatibility. Mat Sci Eng C-Bio S, 2009, 29: 2195-2202

33 Coppens M O. Characterization of fractal surface roughness and its influence on diffusion and reaction. Colloid Surface A, 2001, 187: 257-265

34 Jun C, Penga X F, Lee D J. Diffusion coefficient of Brownian particle in rough micro-channel. J Colloid Interf Sci, 2006, 296: 737-742

35 Hay K M, Dragilab M I, Liburdy J. Theoretical model for the wetting of a rough surface. J Colloid Interf Sci, 2008, 325: 472-477

36 Ren J, Ganapathysubramanian B, Sundararajan S. Experimental analysis of the surface roughness evolution of etched glass for micro/nanofluidic devices. J Micromech Microeng, 2011, 21: 025012

37 Kuhner M, Tampe R, Sackmann E. Lipid mono- and bilayer supported on polymer films: Composite polymer-lipid films on solid substrates. Biophys J, 1994, 67: 217-226

38 Seu K J, Pandey A P, Haque F, et al. Effect of surface treatment on diffusion and domain formation in supported lipid bilayers. Biophys $\mathrm{J}$, 2007, 92: 2445-2450

39 Burlatsky S F, Oshanin G, Cazabat A M, et al. Microscopic model of upward creep of an ultrathin wetting film. Phys Rev Lett, 1995, 76: 86-89

Open Access This article is distributed under the terms of the Creative Commons Attribution License which permits any use, distribution, and reproduction in any medium, provided the original author(s) and source are credited. 


\section{Supporting Information}

Figure S1 Scanning electron micrograph of picoliter-sized reactors.

Figure S2 Schematic drawing of high-throughput pyrosequencing.

Figure S3 Velocity magnitude distribution in the reaction chamber where height represents the depth of microfluidic channel and length denotes a part of its extent. Velocity magnitude is approximately Gaussian distribution.

The supporting information is available online at life.scichina.com and www.springerlink.com. The supporting materials are published as submitted, without typesetting or editing. The responsibility for scientific accuracy and content remains entirely with the authors. 